\title{
Vertical-Cavity Semiconductor Optical Amplifiers (VCSOAs) as optical sensing elements
}

\author{
A.P. Gonzalez-Marcos ${ }^{1}$, A. Hurtado ${ }^{2}$, J.A. Martin-Pereda ${ }^{3}$
}

E.T.S. Ingenieros de Telecomunicación. Universidad Politécnica de Madrid

Ciudad Universitaria 28040. Madrid. Spain

\begin{abstract}
Semiconductor Optical Amplifiers (SOAs) have mainly found application in optical telecommunication networks for optical signal regeneration, wavelength switching or wavelength conversion. The objective of this paper is to report the use of semiconductor optical amplifiers for optical sensing taking into account their optical bistable properties. As it was previously reported, some semiconductor optical amplifiers, including Fabry-Perot and Distributed-Feedback Semiconductor Optical Amplifiers (FPSOAs and DFBSOAs), may exhibit optical bistability. The characteristics of the attained optical bistability in this kind of devices are strongly dependent on different parameters including wavelength, temperature or applied bias current and small variations lead to a change on their bistable properties. As in previous analyses for Fabry-Perot and DFB SOAs, the variations of these parameters and their possible application for optical sensing are reported in this paper for the case of the Vertical-Cavity Semiconductor Optical Amplifier (VCSOA). When using a VCSOA, the input power needed for the appearance of optical bistability is one order of magnitude lower than that needed in edge-emitting devices. This feature, added to the low manufacturing costs of VCSOAs and the ease to integrate them in 2-D arrays, makes the VCSOA a very promising device for its potential use in optical sensing applications.
\end{abstract}

Keywords: Vertical-Cavity Semiconductor Optical Amplifier (VCSOA), Optical Bistability, Optical Sensing

\section{INTRODUCTION}

Usually, in optical sensors, the variation of the optical intensity of a signal gives the information about changes of the parameter (vg temperature) being sensed; this is the case in typical fibers sensors or interferometer devices. Also, we can find commercial applications, even with analog-to-digital conversion, where the analog signal is on the optical domain and depending on its optical intensity, the electrical digital signal generated has changes in frequency from $1 \mathrm{~Hz}$ to $1 \mathrm{MHz}$. With a similar idea in mind, and trying to take advantage of the VCSOA devices, some new approaches have been done with the devices known as VCLAD Vertical-Cavity Laser Amplifier Detectors. In any one of this type of possible sensors, based on photodiodes, there is an inconvenient because the electronic part is beside the sensor device; this is a great disadvantage and the principal problem overcomes with fiber technology sensors.

The idea of using a SOA as optical sensing elements based on bistability has two main advantages with respect to other type of sensors where the information of variability in the parameter that is being sensed it is in the intensity of the optical signal. First one is that we can use the optical sensor far away from the electronics circuits and so we are able to transmit the signal, carrying the information, to the control center. This same type of device can be used to detect several parameters based on the optical output power -optical frequency bistability and optical output power-optical input power bistability. Also, as it works below its threshold, the consuming power is very low and because it is an optical amplifier, the optical signal has enough intensity to reach a further processing unit.

The main requirements for any sensing element is to have enough output contrast for the parameter to be sensed and acting, if possible, in a no intrusive way. The first characteristic allows recognizing small changes in the physical studied parameters. The second one gives the possibility, for the whole system, to work in a regular basis without alterations in its behavior. Moreover, another fact should be of interest. This fact is the possibility to obtain several

\footnotetext{
1 agonmar@tfo.upm.es

2 ahurtado@tfo.upm.es

${ }^{3}$ jamp@tfo.upm.es
} 
output data from a single measure. If the parameters to be analyzed can be obtained in such a way that the obtained result from the sensor clearly depends on several parameters, and they can be independently analyzed, the sensing task would be easier. If a single sensor should allow, for instance, the measure of temperature, optical intensity and optical frequency, from just a single output, this sensor will achieve a higher degree of interest. This is one of the main objectives to be achieved in this works and it will be presented next.

\section{SEMICONDUCTOR LASER AMPLIFIERS BISTABILITY AS SENSING ELEMENT}

Several approaches have been adopted using semiconductor laser amplifiers as sensing elements. For example, Le [1] utilizes the detection of the transparent point of a semiconductor laser amplifier (SLA) for wavelength discrimination. In our previous study on FP-SLA [2], we based our sensing action for wavelength selection on bistability behaviour, as it will be in this work. Some other approaches are reported widely in the literature.

The internal structure of the SLA is very important in order to know tolerance and performance margin. As it is well known, a semiconductor laser amplifier exhibits an strong dependence on the frequency detuning, and it is expected that the input-output power response of the whole structure will

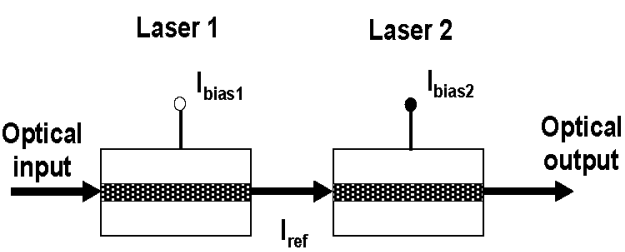

(a)

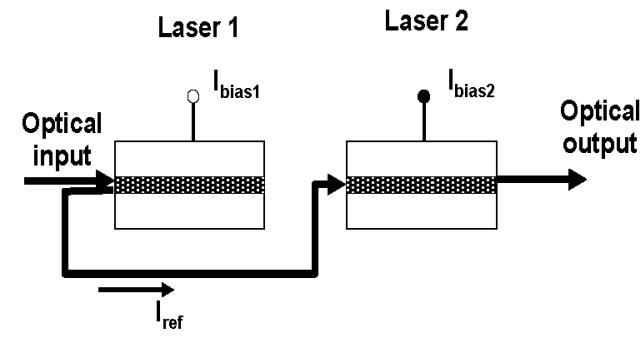

(b)

Fig. 1.- Possible configurations for feedback in cascaded laser structures, (a) with transmitting signal, and (b) with reflecting signal from the first laser.

But to employ optical bistability as a sensing parameter, makes necessary understanding, or at least knowing, the device response for different behaviour conditions. Many studies have been done in DFB, and FP- LAS. We have presented some studies on this direction, with difference approaches, that can be found on references [2,6-7]. Here we concentrate our analysis on the use of VCSOA. On figure 1 we can see different configurations that allow sensing elements as reported in [6]. But before applying these configurations, it is necessary to study the simplest configuration. It is the one represented on fig. 2 for the VCSOA structure. also vary when the frequency of the external signal be modified. When a semiconductor laser is biased just below its threshold, it acts as a resonant-type amplifier. Optical bistability, based on a saturation-induced refractive index change due to external light injection, has been predicted and demonstrated [3]. On the other hand, optical bistability of a semiconductor laser biased above its threshold, and subject to external optical injection has also been observed [4]

(a)
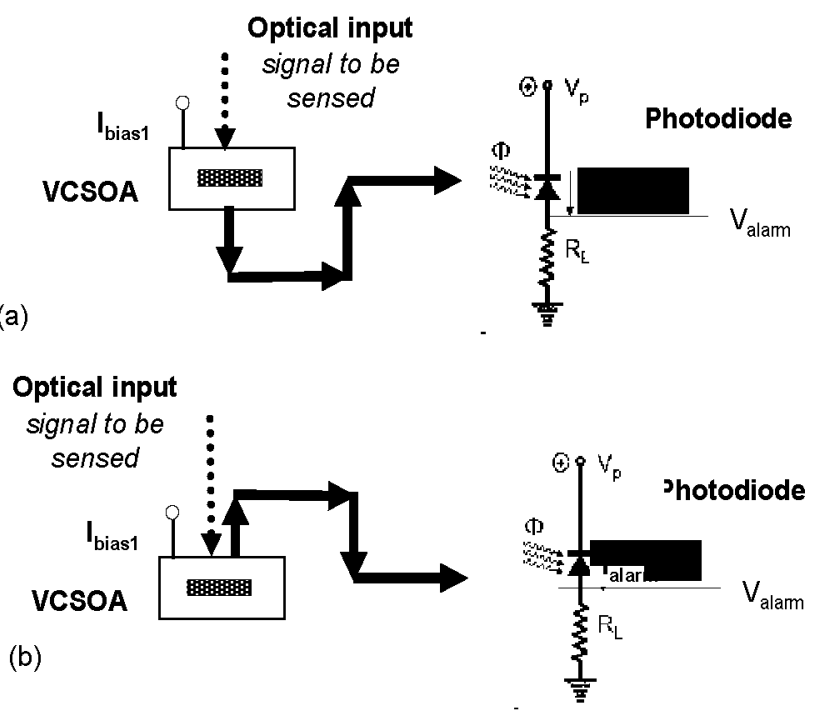

Fig. 2.- Studied configurations for optical amplifiers as sensor elements, (a) with transmitting signal, and (b) with reflecting signal from the VCSOA. 


\section{MODELED VCSOA}

On paper [5], a detailed description of the VCSOA model used in our study it is available. Here we present just the results for a different configuration which allows us to evaluate, in a first approach, how this LD structure can be used as a sensor. Its basic structure appears in Fig. 3. As it may be seen, it is composed by two vertical multilayer gratings with an active layer in between. The effective cavity length covers both, the active layer and a certain number of grating periods. The number of periods in the Bragg reflectors, top and bottom layers, affects to the internal reflectivity achieved by the structure. This reflectivity has an effect on the main behavior of the structure and, as a consequence, on its bistable properties. Hence, it is very important parameter and deserves to be analyzed because sensing properties of the device may be affected by it.

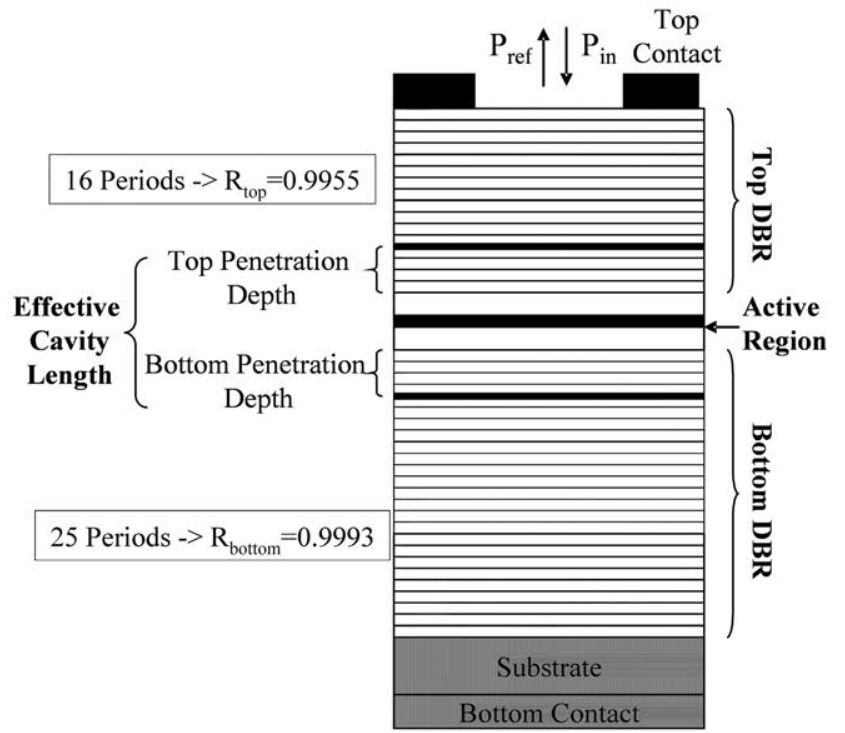

Figure 3.- VCSOA structure.
The equations used in the model for configuration on figure 2 are:

$$
\begin{aligned}
& P_{R}=\frac{\left(\sqrt{R_{t}}-\sqrt{R_{b}} e^{g L_{c}}\right)^{2}+4 \sqrt{R_{t} R_{b}} e^{g L_{c}} \sin ^{2} \phi}{\left(1-R_{t}\right)\left(1+R_{b} e^{g L_{c}}\right)\left(e^{g L_{c}}-1\right)} I_{a v} P_{y} g L_{c} \\
& P_{T}=\frac{\left(1-R_{b}\right) g L}{\left(1+R_{b} e^{g L}\right)\left(1-e^{-g L}\right)} P_{y} I_{a v}
\end{aligned}
$$

where $R_{t}$ and $R_{b}$ are the top and bottom DBR reflectivity, $L_{c}$ is the effective length of the VCSOA, $g$ is the gain per unit length and $\phi$ is the single-pass phase change. $P_{x}$ and $P_{y}$ are scaling parameters

\section{DISCUSSION OF VCSOA AS SENSING ELEMENT}

The behavior of the VCSOA has been studied for four critical parameters:

- Bias current (analysis in percentage with respect to the threshold current, being this current always below threshold)

- Frequency detuning (difference between optical proper frequency of the VCSOA and the impinging signal)

- Optical input power

- $\quad$ Number of periods of the top DBR -Distributed Bragg Reflector.

The bistability behaviors to be reported here analyze frequency detuning and optical input power. This means that we will be able to sense optical wavelength and optical power.

One of the new characteristics to be reported in our present results is that this structure has been analyzed for reflection and for transmission configurations. Both of them are shown in fig. 2. It is obvious that figure 3 does not represent the structure for transmission. But the results obtained here can be considered a good approach as, in any case, it has been taken in account how are designed the upper and bottom contacts to introduce the bias current. 


\subsection{Frequency domain}

As it has being reported in [5], the theoretical analysis of DRB has been done as in a Fabry-Perot cavity where its cavity length depends on the number of period existing at the bottom and top DBR. This is because they may be considered as equivalent to the mirrors of a FP resonator. In this way the longitudinal modes interval is always $\pi$ and, remembering the well know relation,

$\Delta f=\frac{c}{2 L n} ; \quad \Delta \lambda=\frac{\lambda^{2}}{2 L n}$

where,

$\mathrm{n}=3.2$ (refractive index)

$\mathrm{L}=1.56 \mu \mathrm{m}$ (effective cavity length; see [5])

$\mathrm{C}=3 \cdot 10^{8} \mathrm{~m} / \mathrm{s}$ (light speed in vacuum )

We have that $\Delta \mathrm{f} \approx 30 \mathrm{Thz}$ corresponds to $\pi$. Then $10^{-4} \pi$ is approximately $3 \mathrm{Ghz}$. Having in mind these values, they will help us to understand the next figures and how it is possible to evaluate the precision level of the sensor to be developed and proposed by us.

For the first analyzed structure we have fixed the number of periods at the upper DBR in 16 for the VCSOA acting as sensor; as bottom period is 25 , the output is maximized for the reflecting signal. This means that the transmitted signal will have less power than the reflected one. Let us see what happens in these two cases:

a) We have studied first how the sensor responds to wavelength variations when we change the bias current. The signal to be sensed is also fixed to an optical power of $25 \mu \mathrm{W}$. This will allow us to see the tolerance to bias current and know if we can design the device for different applications. The bias current is represented on its relation to the threshold
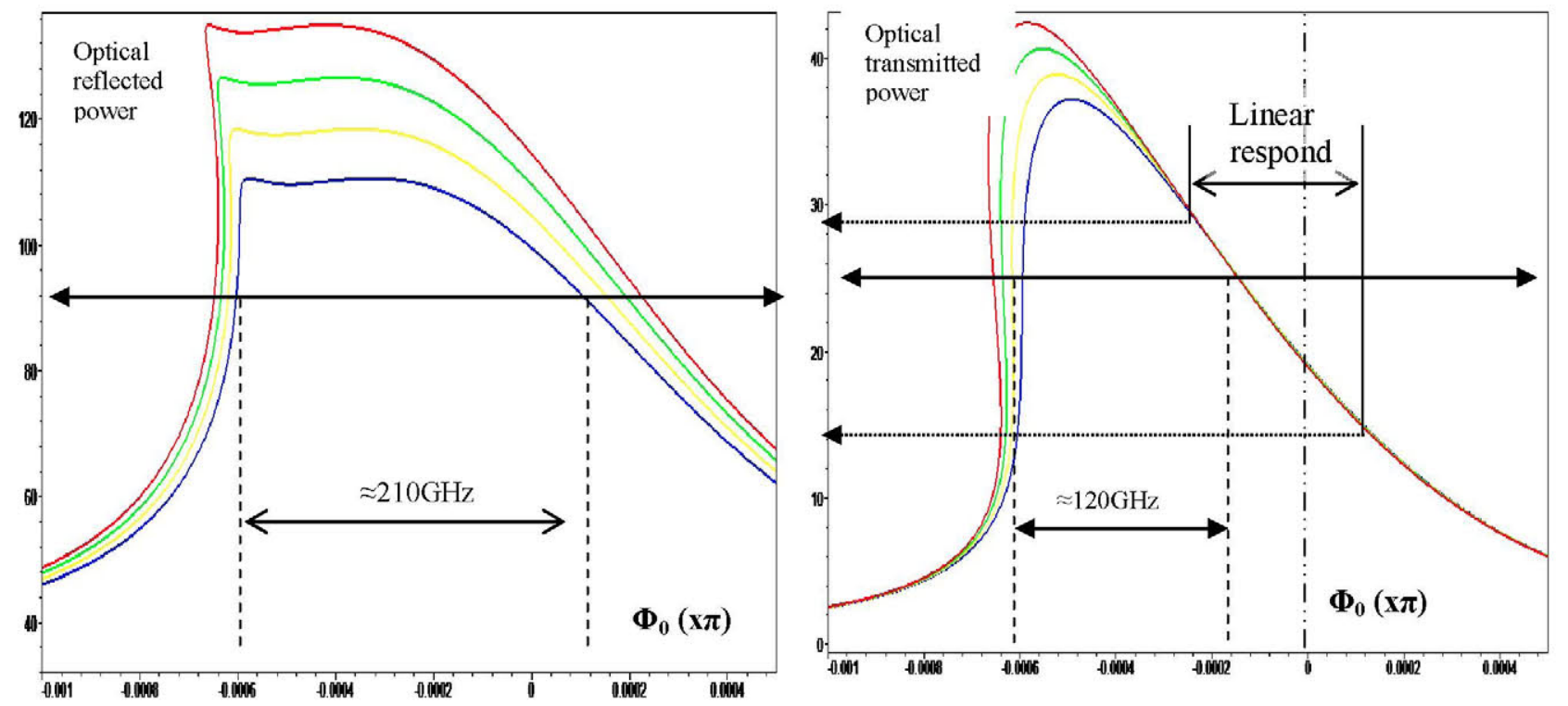

Figure 4.- Optical output in $\mu \mathrm{W}$ versus frequency detuning as a factor of $\pi$. With the parameter used $0,0001 \pi$ is $\approx 3 \mathrm{GHz}$ frequency detuning goes from $30 \mathrm{GHz}$ to $4 \mathrm{GHz}$. Left graph correspond to reflected signal (configuration (b) on figure 2); right graph is for the transmitted signal, fig2(a)

current that as we said is always below this value. As a consequence we never arrive to a bias current $100 \%$ of the threshold value. On figure 4 it is represented the optical output power versus frequency detuning for a bias current of $99 \%, 98 \%, 97 \%$ y $96 \%$ the threshold current applied to the VCSOA.

We observe that at higher bias current the curves are more asymmetric and they are shifted to smaller frequencies. The reason is because as higher the bias current is, the higher is the no-saturated gain coefficient $\mathrm{g}_{0}$, being $\mathrm{g}_{0}=\mathrm{a}\left(\mathrm{n}-\mathrm{n}_{0}\right)$ and a 
the linear gain coefficient of the material, $n$ the carrier density and $\mathrm{n}_{0}$ the transparency carrier density. When the nosaturated gain coefficient is higher, the injected external optical signal allows a greater recombination; this makes an increasing in the refractive index of the active region. The change produced in the refractive index decreases the

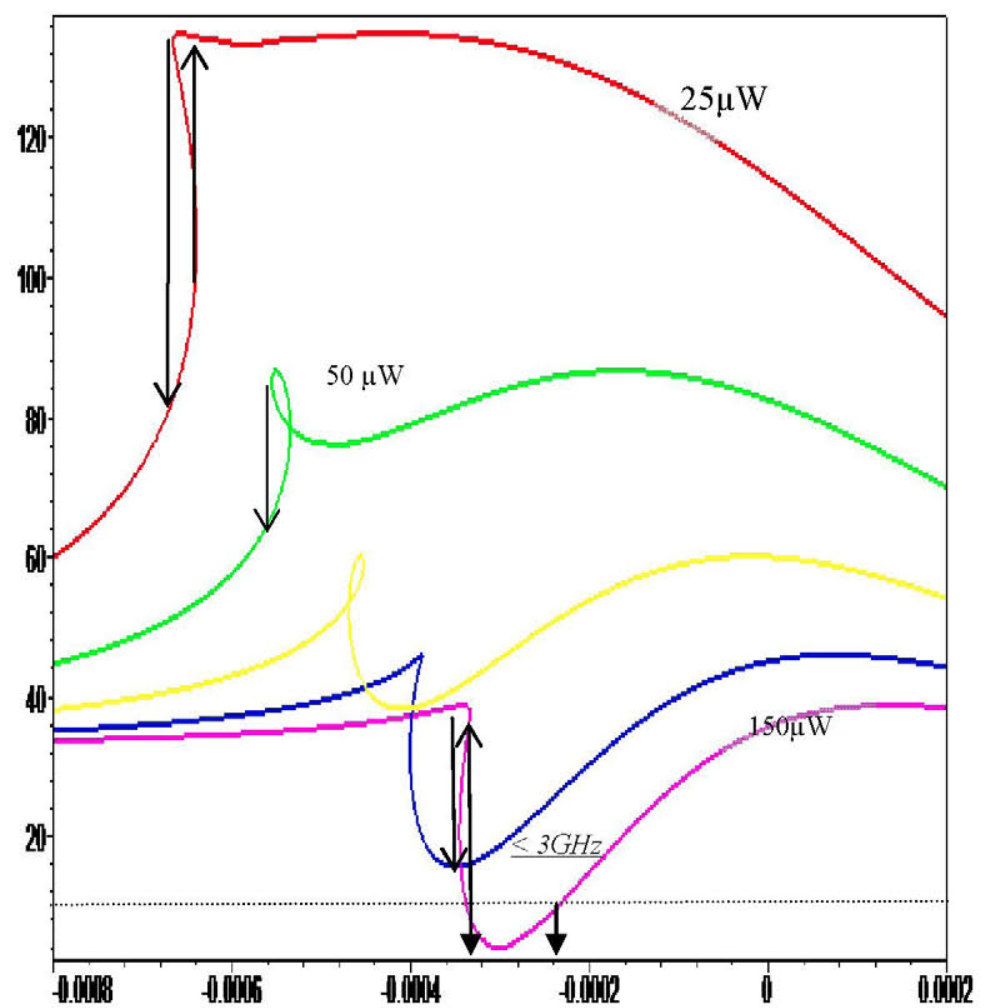

Figure 5.- Optical output in $\mu \mathrm{W}$ versus frequency detuning as a factor of $\pi$, for different optical injected power. With the parameter used $0,0001 \pi$ is $\approx 3 \mathrm{GHz}$ frequency detuning goes from $24 \mathrm{GHz}$ to $6 \mathrm{GHz}$. Reflected configuration. resonant longitudinal mode of the VCSOA.

On figure 4 we can not see a clear bistability loop because in a VCSOA it is needed to be working very near to the threshold current to see it. This is due to the very small active region of the VCSOA compared to other structures.

b) The analisys of how the sensor responds to wavelength variations when we change the external optical power impinging, this will allow to see the tolerance to optical power of the signal to be sensed. In this case, the bias current is fixed to $99 \%$, very near of threshold current, in order to see more clearly the presence of bistability. In every case, with transmitted and reflected configuration, the loop is anticlockwise.

The optical power incident to the device has been taking with relative high values, namely from $25,50,75,100$ to $150 \mu \mathrm{W}$, in order to evaluate where the bistability loop appears more clearly defined. We can see the presence of a clear gap under $10 \mu \mathrm{W}$ of less than $3 \mathrm{GHz}$, but it is needed to have an input power very high of $150 \mu \mathrm{W}$. On reflected configuration near the resonant frequency there is an small area with

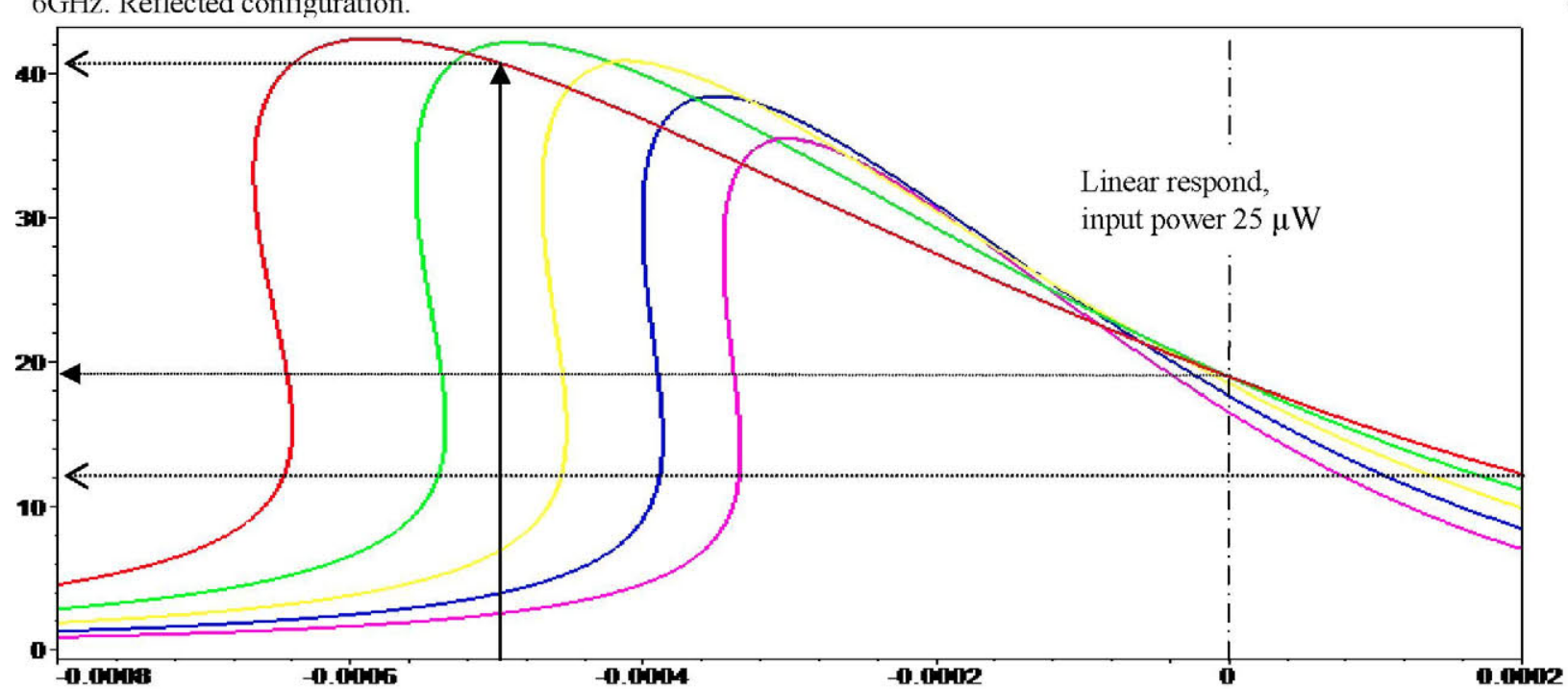

Figure 6.- Optical output power in $\mu \mathrm{W}$ versus frequency detuning as a factor of $\pi$, for different optical impinging power. With the parameter used $0.0001 \pi$ is $\approx 3 \mathrm{GHz}$ frequency detuning goes from $24 \mathrm{GHz}$ to $6 \mathrm{GHz}$. Transmitted configuration. 
linear respond that may used but it is more interesting to use the transmission configuration where we have a linear respond that cover a wide range $\approx \pm 15 \mathrm{GHz}$. On fig. 6 we have a better view of the linear respond indicated on fig.4(b); here we can see that it is better to work with less optical power on the signal sensed in order to have a large range of values for detecting detuning or changes in the optical frequency. Looking on fig. 6 we obtain an equation:

$$
P_{\text {out }}(\mu W)=-4,1428 \times\left(x .10^{4}-4,6\right)
$$

with $\mathrm{x}$ equal to the frequency detuning $\mathrm{x} \pi$; we have to remember that $\mathrm{x}=10^{-4}$ corresponds, in the modelled device to $3 \mathrm{GHz}$. We can reduce the value of $\mathrm{x}$, but to obtain its limit it must be studied the stability device performance. But theoretically seems that we have a wavelength shift sensor with a high accuracy.

\subsection{Input power domain}

Another important parameter is the optical power of an optical signal, besides the possibility of being amplified; it could be interesting to have a sensor of small variations of power. In this section, we will show the characteristic behavior of the proposed sensor device when we can bee sure that the monitor signal has no change its frequency.

First we represent the output power versus input power, on reflected and transmitted configurations, in similar cases as the previously studies. The top resonant DBR has 16 periods.

A) Initial phase detuning equal to $-5 \cdot 10^{-4} \pi$. Bias current equal to: $99.5 \%, 99 \%, 98.5 \%, 98 \%, 97.5 \%$ y $97 \%$ of threshold value.
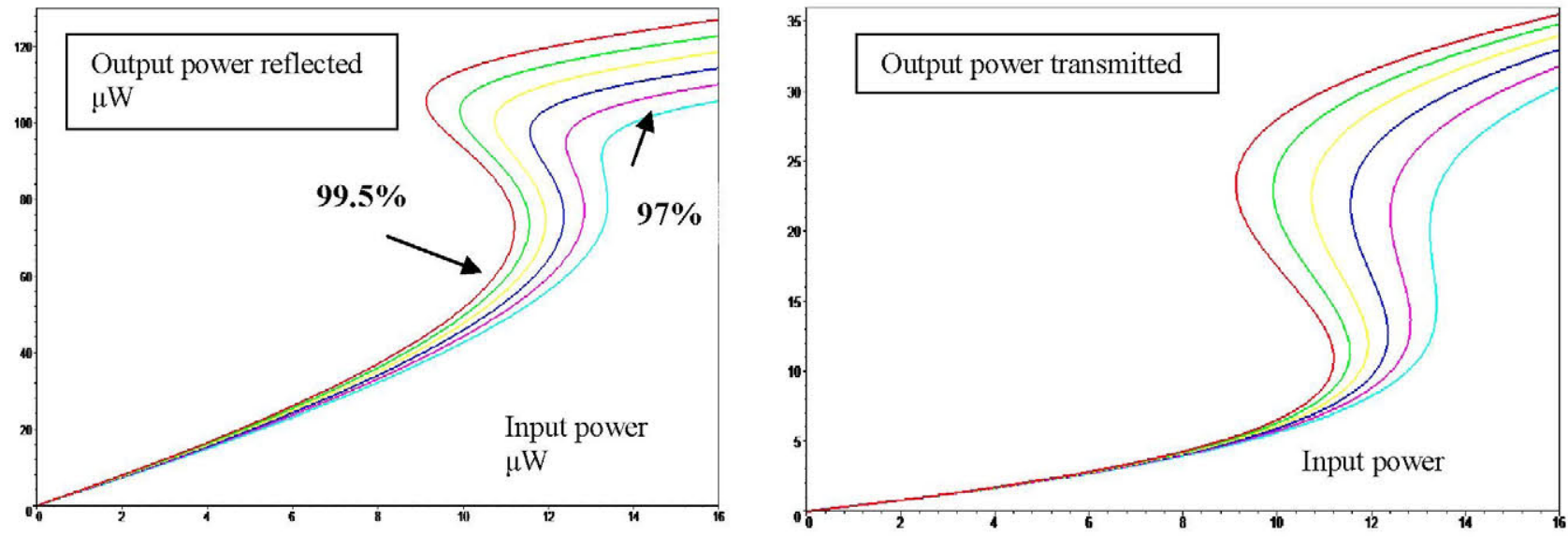

Figure 7.- Output power $(\mu \mathrm{W})$ versus optical power of impinging signal in $(\mu \mathrm{W})$. Left curve output reflected signal, right graph transmitted optical signal. The curves correspond to different bias current

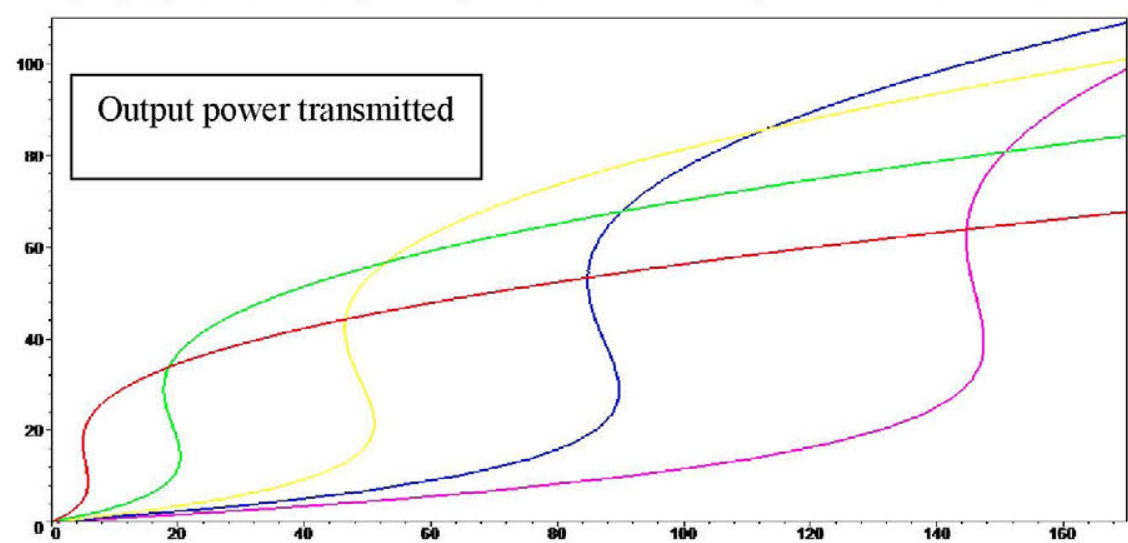

Figure 8.- Output power $(\mu \mathrm{W})$ versus optical power of impinging signal in $(\mu \mathrm{W})$, for several initial phase detuning: $-4,-6,-8,-9.5 \mathrm{y}-11 \cdot 10^{-4} \pi$.
As we can see in fig. 7 there is not too much change in both plots, so let see what happen if the impinging signal has a different frequency.

B) Initial phase detunning: $-4,-6$, $-8,-9,5$ y $-11 \cdot 10^{-4} \pi$.

Bias current: $99 \%$.

On fig. 8 we represent the output transmission power, but as we can see there is nothing relevant for the application we are working in. Just to comment that as greater is 
the initial detuning a higher power for switch it is needed.

Nevertheless, if we look the reflected power we find that different bistabilities loops appear. Fig. 8.a it is a syntesis of all the types of bistability loops that can be found. It can be observed that as the detuning is increased the bistability

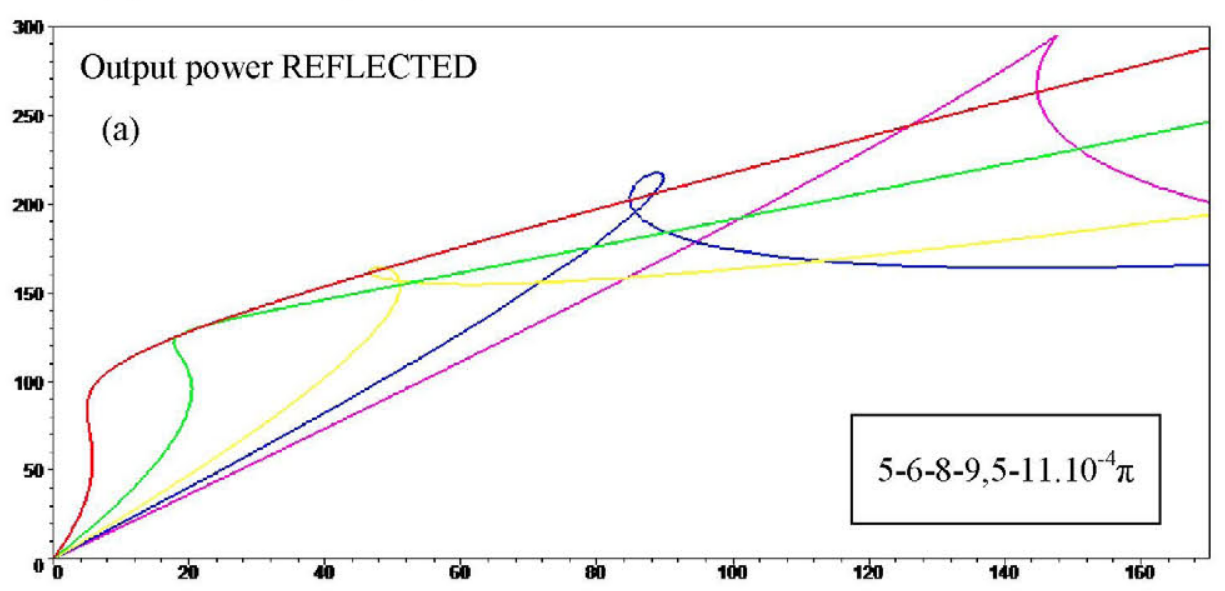
changes from the classical anticlockwise bistable loop to the clockwise bistable loop, with the intermediate $\mathrm{X}$-shape or butterfly bistable loop.

A more detail discussion of this effect can be found on reference [5] and a more obvious application on reference [8]. Fig. 8.b shows the bistability behaviour for small detuning which need less input power for
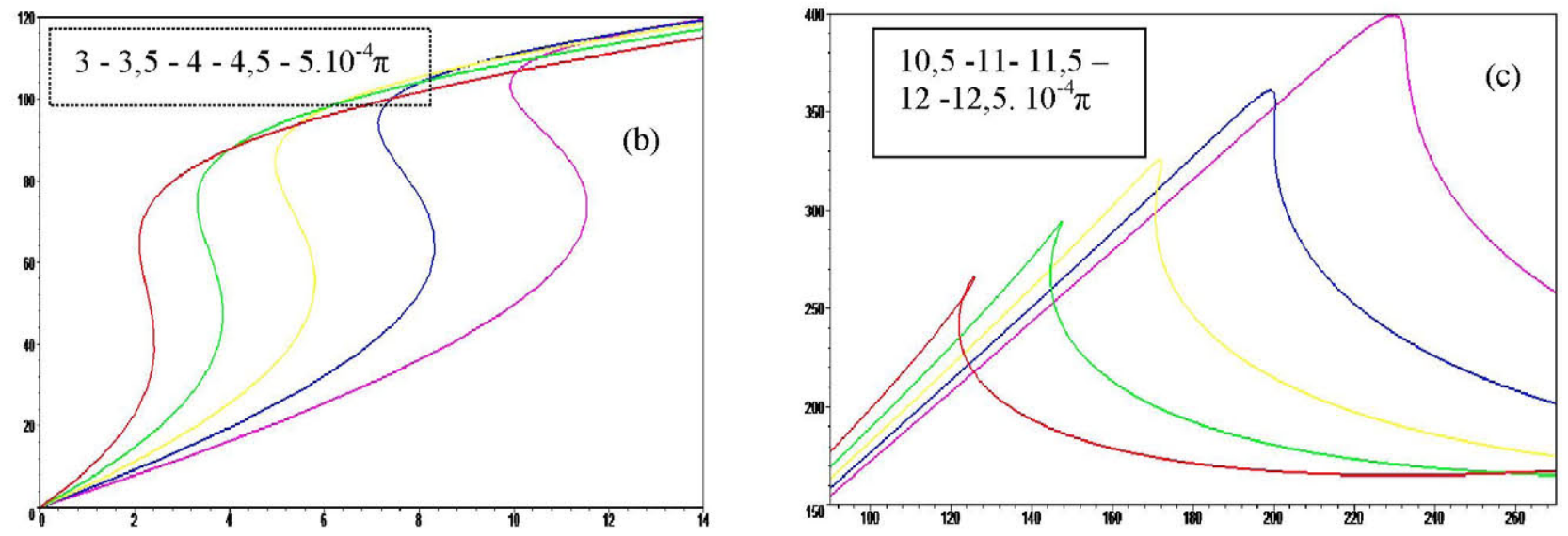

Figure 9.- Output power $(\mu \mathrm{W})$ versus optical power of impinging signal in $(\mu \mathrm{W})$, (a) Wide range of detuning, (b) a detail for small detuning and input power from $0-14 \mu \mathrm{W}$ for several initial phase detuning, and (c) a detail for higher detuning and input power from $90-260 \mu \mathrm{W}$.

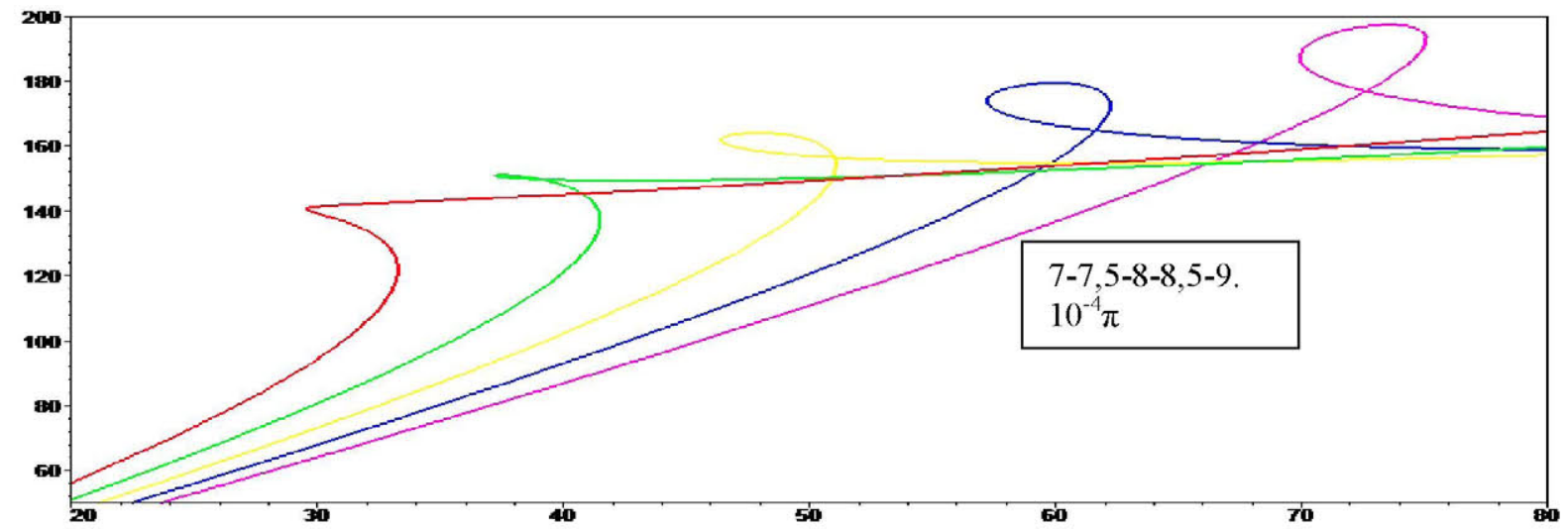

Figure 10.- Output power $(\mu \mathrm{W})$ versus optical power of impinging signal in $(\mu \mathrm{W})$, for several initial phase detuning: input power range from $20-80 \mu \mathrm{W}$ 
switching. On the other side Fig.8.c shows that for higher detuning the switching power is higher. Fig. 9 shows what happens in between the two types of bistability loops, and how it changes anticlockwise bistable loop to the clockwise bistability.

As the objective of this work is to analized the possibility of employing the VCSOA with bias polarization near and below threshold as a sensor device, let us show how it is the behaviour when the bias current change for the three different bistable loops obtained on the reflected configuration.

C) In this case we maintain the same parameters as in A) of this section. We represent output power versus input power with the upper resonant DBR has 16 periods, but we only study reflected configuration. On figure 10 the four plots have shown how the influence of frequency detuning and bias current is. Each plot corresponds to a different detuning and it can be seen that if we want not to have a small switching and a wide hysteresis cycle then we must apply a bias current far below the threshold value.

The bias current studied varies from $99.5 \%, 99 \%, 98.5 \%, 98 \%, 97.5 \%$ and $97 \%$ of threshold value. Initial phase detuning studied is indicated in each plot and they were chosen from results of figure 9 and 10 .
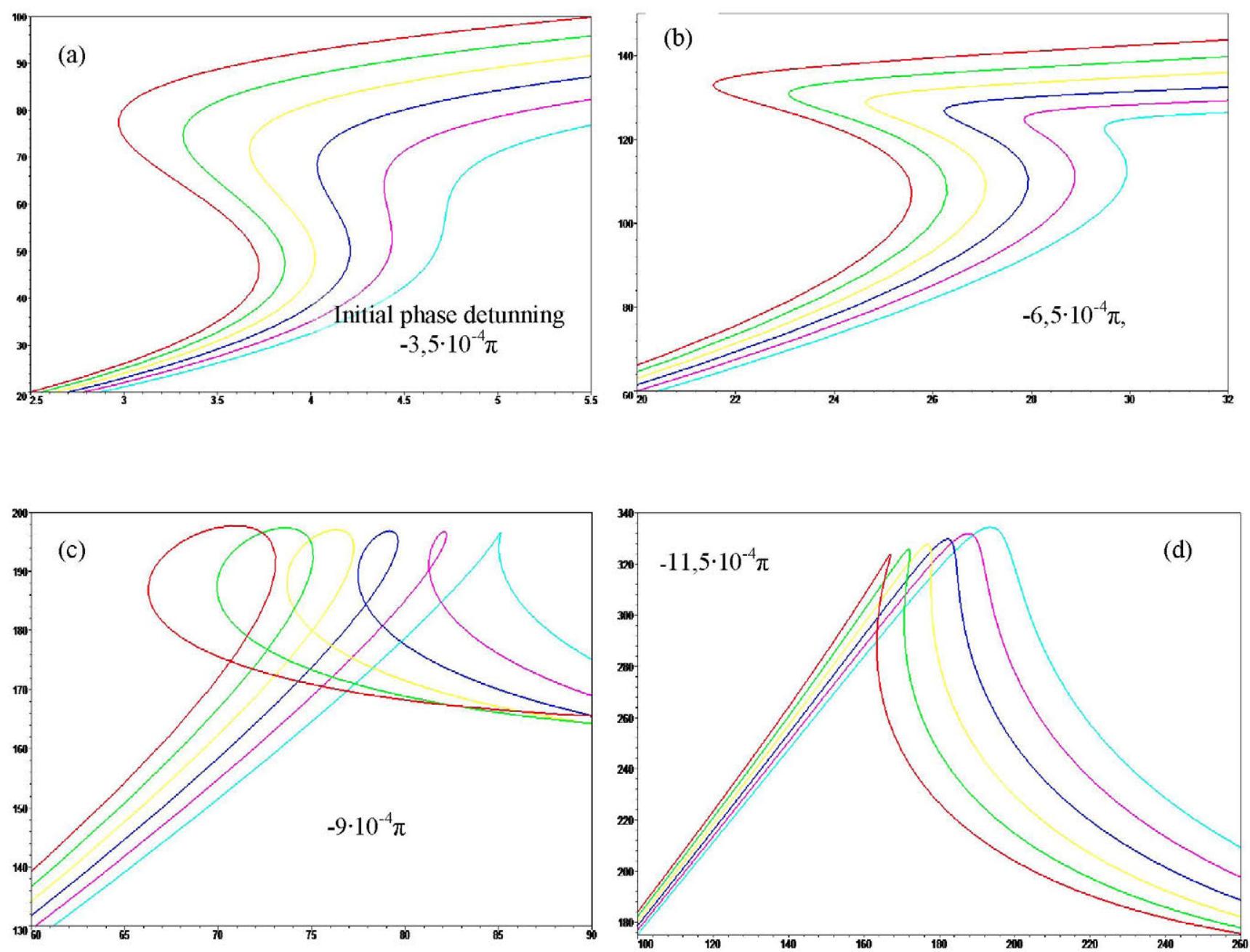

Figure 11.- Output power $(\mu \mathrm{W})$ versus optical power of impinging signal in $(\mu \mathrm{W})$, for several initial phase detuning:

(a) input range: $2,5-5,5 \mu \mathrm{W}$

(b) input range: $20-32 \mu \mathrm{W}$

(c) input range: $60-90 \mu \mathrm{W}$

(d) input range: $100-250 \mu \mathrm{W}$ 


\section{Results for different VCSOA based on number of period on DBR}

Finally we present a brief study on how affects the device characteristic when the design of the VCSOA changes. On previous figures we used an upper period of DBR equal to 16. This value gives more output power for the reflected signal than for transmitted signal. On results presented on figure 13, and 14 we show the main plot of previous sections with and input power equal to $25 \mu \mathrm{W}$ for the detuning sense and an initial phase detuning equal to $-3 \cdot 10^{-4} \pi$ for the input power variation. In both cases the bias current is $99,5 \%$ the threshold current.
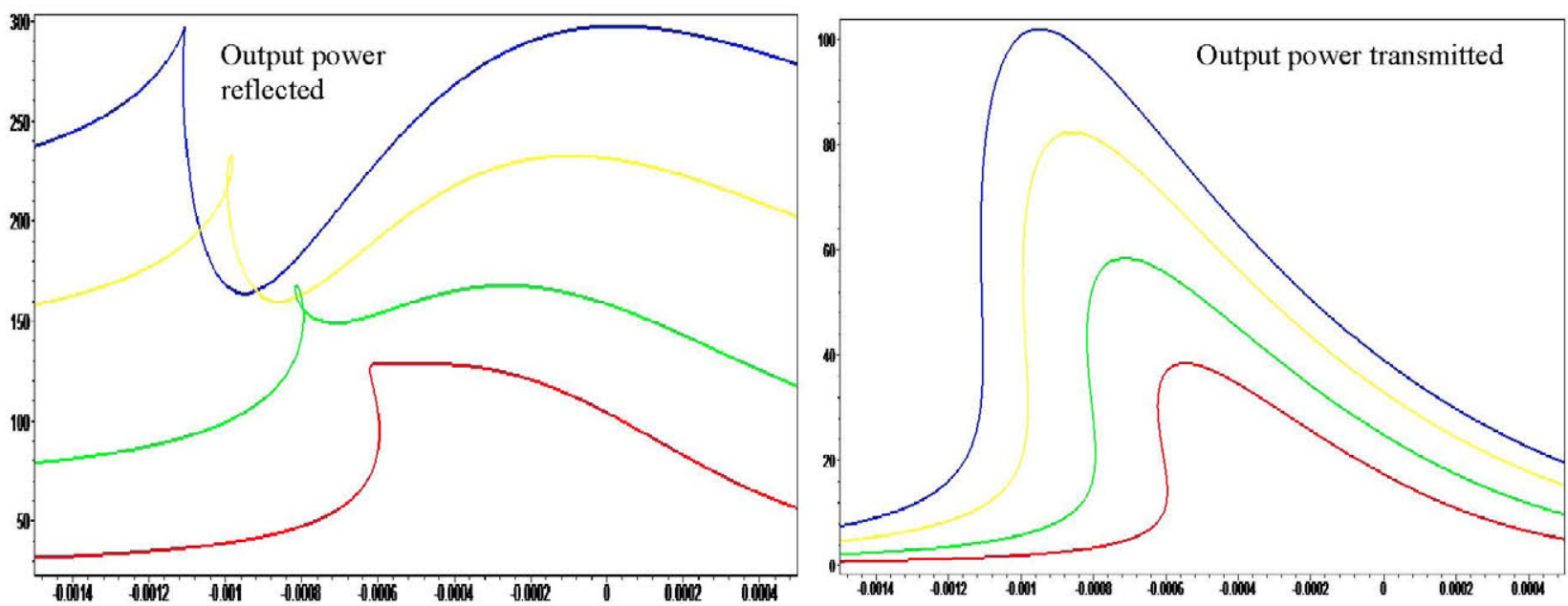

Figure 12.- Optical output in $\mu \mathrm{W}$ versus frequency detuning as a factor of $\pi$. With the parameter used $0,0001 \pi$ is $\approx 3 \mathrm{GHz}$ frequency detuning goes from $44 \mathrm{GHz}$ to $9 \mathrm{GHz}$. Several top DBR periods: $16-17-18-19$
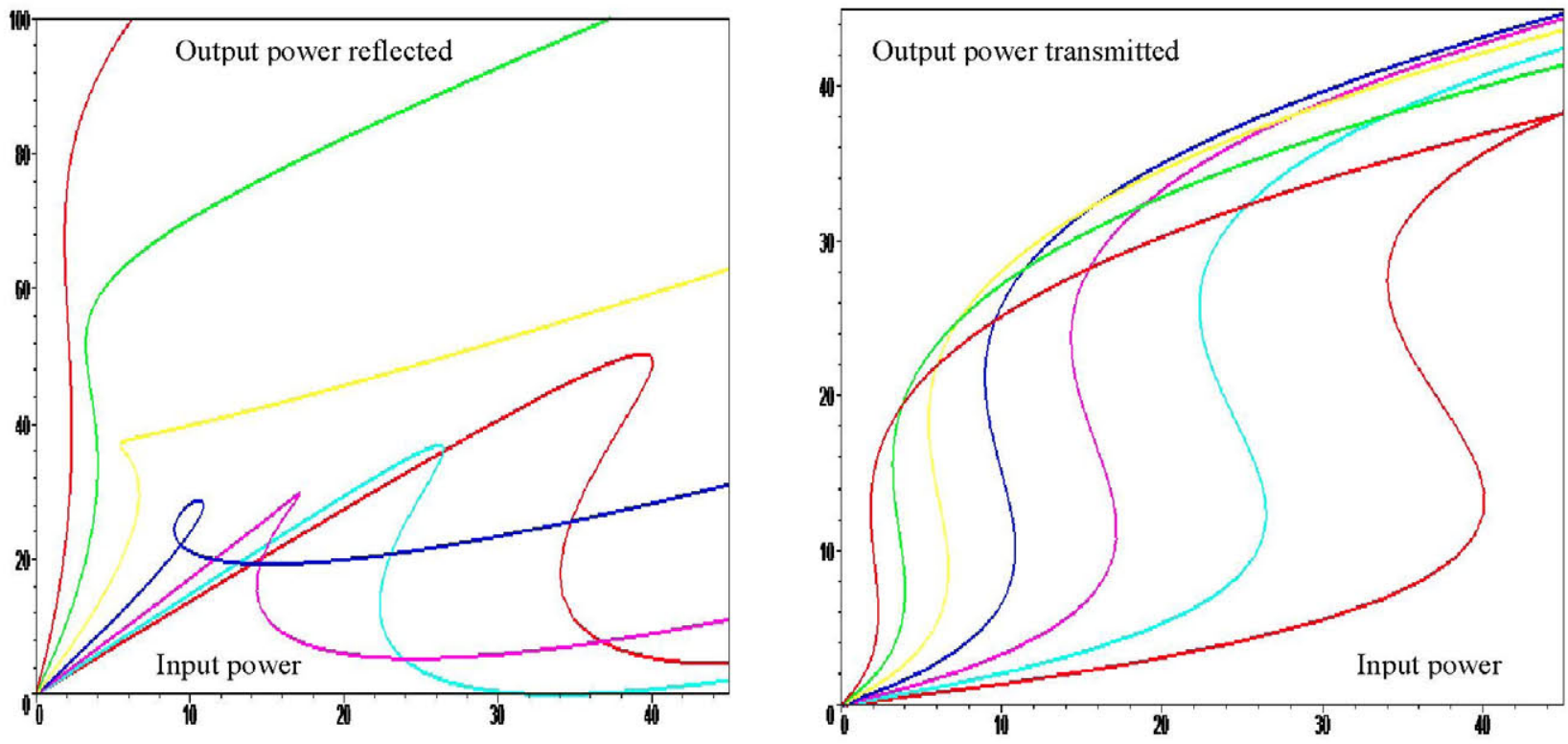

Figure 13.- Output power $(\mu \mathrm{W})$ versus optical power of impinging signal in $(\mu \mathrm{W})$. Input power from $0-50 \mu \mathrm{W}$. Top DBR periods are equal to $16,17,18,19,20,21$ y 22.

From these figures it can be seen, as can be expected, that as higher the number of periods is, the output power obtained is greater. Due to this result, the linear response area will be larger. This, from the point of view of using this device as a sensor, can be an advantage. Also we can observe the same effect on transmitted power versus input power when the number of periods is higher. The number of periods affects the internal reflectivity so it will change the longitudinal resonant mode, and this may cause that the output power would be less on transmission or in reflections. In any case both responds are complementary for the same values. 


\section{CONCLUSIONS}

The reported results are just a summary of the several possibilities offered by this kind of configuration. As it has been shown, several of the more important parameters in any communication system, namely, optical frequency and optical power intensity, are possible to be sensed with a configuration as the one here reported. The importance of this fact is evident. Similar devices and components as the usually employed in any communication system may now be employed as sensing elements. If we compare the degree of precision achieved with this method, with other more common techniques, we see that the results are much better here than with other methods. This allows us to consider this reported technique as a potential candidate to be employed in future sensing systems.

\section{ACKNOWLEDGEMENTS}

This work was partly supported by CICYT, grant TIC2003-04309, and CAM "Comunidad Autónoma de Madrid", grant FPIFormación Personal Investigador.

\section{REFERENCES}

1. San-Liang Lee "Wavelength measurement and tracking using semiconductor laser amplifiers for applications in photonic networks" Photonics Technology Letters, IEEE Volume: 10, Issue: 3, 439-441, Mar 1998

2. Hurtado, Ana Gonzalez-Marcos, Jose A. Martin-Pereda "Wavelength monitoring with semiconductor laser amplifiers" Proc. SPIE Vol. 5502, p. 508-511, Second European Workshop on Optical Fibre Sensors; Jose M. Lopez-Higuera, Brian Culshaw; Eds. Jun 2004

3. K. Otuska, H. Iwamura, IEEE J. Quantum Electron. 19 (1983) p.1184; Otuska, H. Iwamura, Electron. Lett. 19 (1983)p. 262; M.J. Adams, IEE Proc. Pt. J. 132 (1985) p.343; M.J. Adams, H.J. Westlake, M.J. O’Mahony, I.D. Henning IEEE J. Quantum Electron. 21 (1985) p. 1498; H. Kawaguchi, IEEE J. Quantum Electron. 23 (1987) 1429 .

4. H. Kawaguchi, K. Inoue, T. Matsuoka, K. Otsuka, IEEE J.Quantum Electron. 21 (1985) 1314.; J.M. Liu, H.F. Chen, T.B. Simpson, IEEE Photon. Technol. Lett. 9 (1997) 1325.

5. A. Hurtado, A. Gonzalez-Marcos, J.A. Martin-Pereda, 'Modeling Reflective Bistability in a Vertical-Cavity Semiconductor Optical Amplifier (VCSOA)', IEEE Journal of Quantum Electronics, Vol. 41, No. 3, pp. 376383. March 2005

6. Ana P. Gonzalez-Marcos, Jose A. Martin-Pereda, Antonio Hurtado "Laser diode bistability as sensor of optical signal parameters" Proc. SPIE Vol. 5579, p. 152-159, Photonics North 2004: Photonic Applications in Telecommunications, Sensors, Software, and Lasers; John C. Armitage, Roger A. Lessard, George A. Lampropoulos; Eds. Nov 2004

7. A. P. Gonzalez-Marcos, A. Hurtado, J. A. Martin-Pereda "Optical bistable devices as sensing elements" Proc. SPIE Vol. 5611, p. 63-70, Unmanned/Unattended Sensors and Sensor Networks; Edward M. Carapezza; Ed. Nov 2004

8. A. Hurtado, A. Gonzalez-Marcos, J.A. Martin-Pereda "Low-Power Vertical Cavity NAND Gate" paper $\mathrm{n}^{\circ}$ 5840_59. Photonic Materials, Devices, and Applications EMT05 Microtechnologies for the New Millennium 2005. 